\title{
Ribosomal protein gene transcription in Saccharomyces cerevisiae shows a biphasic response to nutritional changes
}

\author{
Gerard Griffioen, Ruud J. Laan, Willem H. Mager and Rudi J. Planta \\ Author for correspondence: Rudi J. Planta. Tel: +31204447548. Fax: +31204447553. \\ e-mail: brink@chem.vu.nl
}

Department of Biochemistry and Molecular Biology,

IMBW, BioCentrum

Amsterdam, Vrije

Universiteit, de Boelelaan

1083, 1081 HV Amsterdam,

The Netherlands

\begin{abstract}
Nutrients are major determinants of ribosomal protein (rp-) gene transcription in Saccharomyces cerevisiae. In order to investigate the molecular mechanisms underlying this nutritional control, yeast mutants that display defects in the glucose upshift response of rp-gene transcription were isolated. Interestingly, although growth of these mutants on glucose-containing medium was severely affected an initial increase in rp-gene transcription by nutritional upshift was still observed. However, at later time points, rp-mRNA levels decreased strongly. Various other types of severe growth limitation also did not prevent the initial upshift in transcription. The results suggest that the glucose upshift response of rp-gene transcription comprises two phases: an initial, transient response independent of the actual growth potential, and a sustained response which is dependent on growth and requires both glucose and adequate nitrogen sources. Previously, it was found that protein kinase $A$ (Pka) mediates the initial upshift response, without the need for regulation of Pka activity by CAMP. The present data substantiate that, besides the RAS/adenylate cyclase pathway, an alternative pathway through Pka regulates rp-gene transcription. In addition, evidence is presented that the sustained response does not require Pka activity. Based on these results, taken together, a model is proposed in which rp-gene transcription is dynamically regulated by multiple signal transduction pathways.
\end{abstract}

Keywords: Saccharomyces cerevisiae, nutritional signalling, ribosomal protein gene

\section{INTRODUCTION}

Sensing of nutrients is an essential property of microbial cells in order for them to adapt adequately to variations in growth conditions. For instance, Saccharomyces cerevisiae cells cultured under optimal growth conditions display a high rate of protein biosynthesis (Kief \& Warner, 1981) and divide rapidly. This is in contrast with yeast cells entering stationary phase, when nutrients essential for growth, such as the nitrogen or carbon source, become limiting. In order to survive such conditions, haploid yeast cells arrest in $G_{1}$ of the cell cycle and enter a quiescent state called $G_{0}$, whereas diploid cells will sporulate (reviewed by Werner-Washburne et al., 1993).

In $S$. cerevisiae several signal transduction pathways

Abbreviations: FGM, fermentable growth medium; Pka, protein kinase A; rp-gene, ribosomal protein gene. involved in nutritional regulation of metabolic processes, the cell cycle and sporulation have been identified. The best-studied of these so far is the main glucose repression pathway. In fermenting yeast cells, this pathway is responsible for the repression of genes involved in mitochondrial respiration, gluconeogenesis, the glyoxylate cycle and catabolism of alternative sugars (Gancedo, 1992; Trumbly, 1992). Another example is the pathway for general amino acid control. When yeast cells are starved for amino acids, genes involved in the anabolism of amino acids are activated through this pathway (Hinnebusch, 1988, 1994).

It has been established that protein kinase A ( $\mathrm{Pka}$ ) activity plays an important role in regulation of many growthrelated processes, such as progression through $G_{1}$ of the cell cycle, regulation of enzyme activities involved in energy mobilization, and the transcriptional control of several stress-related genes (reviewed by Broach, 1991; Thevelein, 1992). Recently, evidence has been presented 
that $\mathrm{Pka}$ activity in yeast is regulated in at least two different ways. Firstly, Pka activity is modulated by the RAS/adenylate cyclase pathway through cAMP (Toda et al., 1985, 1987a). Catalytically inactive Pka consists of a tetramer of two regulatory subunits and two catalytic subunits (Toda et al., 1987b). Binding of cAMP to the regulatory subunits causes the release of active catalytic subunits. Although the function of the RAS/adenylate cyclase pathway is not completely clear, mutations altering its activity lead to many distinct phenotypes. Constitutive activation or deactivation of this pathway leads to defective regulation in the nutritional control of the cell cycle, the synthesis of storage carbohydrates, sporulation of diploids, post-translational modification of enzymes and transcription of stress-related genes (Belazzi et al., 1991 ; Broek et al., 1987; Kataoka et al., 1984; Marchler et al., 1993; Matsumoto et al., 1983a, b; Tanaka et al., 1988; Toda et al., 1985; see Thevelein, 1994, for a recent review). In addition, it has recently been demonstrated that $\mathrm{Pka}$ activity is also regulated in a cAMP-independent fashion (Thevelein, 1991, 1992, 1994). Activation of Pka through this alternative pathway is only accomplished in the presence of a complete growth medium supplemented with a fermentable carbon source. Therefore, this pathway is designated the Fermentable Growth Medium (FGM)induced pathway. The FGM pathway may further increase the activity of free catalytic subunits of $\mathrm{Pka}$ and may be involved in a number of processes which were found to be regulated by $\mathrm{Pka}$, but not exclusively through the RAS/adenylate cyclase pathway. For instance, Cameron et al. (1988) observed that yeast mutants having a low constitutive activity of $\mathrm{Pka}$ respond normally to nutrient limitation. This is also true for the regulation of trehalase activity and for derepression of CTT1 transcription (Belazzi et al., 1991; Durnez et al., 1994; Hirimburegama et al., 1992).

In this paper we present results of an analysis of the regulation of ribosomal protein (rp-) gene transcription. Transcription of such genes is correlated with the growth conditions in order to adjust the protein biosynthetic capacity to the physiological demands (Donovan \& Pearson, 1986; Herruer et al., 1987; Kraakman et al., 1993; Mager \& Planta, 1991). This is well demonstrated by performing a glucose upshift experiment, in which a fermentable amount of glucose is added to a respiring yeast culture. This switch of carbon source leads to a strong coordinate activation of rp-gene transcription. A similar activation of transcription is observed when nitrogen is added to nitrogen-starved yeast cells (Goncalves et al., 1995; Neuman-Silberberg et al., 1995). Therefore, a better understanding of the regulation of $\mathrm{rp}$ gene transcription will increase our knowledge of nutrient sensing mechanisms and the signal transduction pathways triggered by nutrients in $S$. cerevisiae. Recently, we have identified several mutants displaying defects in nutritional regulation of rp-gene transcription and have established, in agreement with results obtained by others, that Pka activity plays an important role in the glucose upshift response of rp-gene transcription (Griffioen t al., 1994; Klein \& Struhl, 1994). In the work described here we have extended these studies and we demonstrate that rpgene transcription shows transient and sustained responses to nutritional changes. Furthermore, we found that at least three different nutritional signal transduction pathways are involved in generating these responses.

\section{METHODS}

Yeast strains and growth conditions. Strain CH2881-1D (MATa leu 2 ura 3 ade 2 trp 1 lys $2:: d$ ) (Fleig et al., 1986; a kind gift of P. Philippsen) was used to construct strain CPL25 (see below). Strains Y153 (MATa leu2-3,112 ura3-52 trp1-901 bis3-

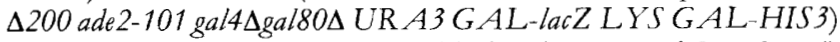
(Durfee et al., 1993), JT100 (MATa leu2 bis3 trp1 ade 8 ura3 can1) (a kind gift of J. Thevelein, K.U. Leuven, Belgium), RG7M1 (Goncalves et al., 1995) and SGP406 (MATa leu2-3,112 trp1 ura3-52 bis3 tpk1::URA3 tpk2::HIS3 tpk3::TRP1 yak 1::LEU2) (Garrett \& Broach, 1989) were used in the glucose upshift and nitrogen refeeding experiments, as indicated in the legends to the figures.

Cells were grown in YNB $(0 \cdot 7 \%, w / v$, yeast nitrogen base), supplemented with $2 \%(\mathrm{w} / \mathrm{v})$ glucose (unless otherwise indicated) and appropriate amino acids and purines/pyrimidines. A glucose upshift experiment was performed as follows. Yeast cells were pre-grown on YNB/glycerol $(0.7 \%$ YNB plus $5 \%$, $\mathrm{w} / \mathrm{v}$, glycerol, $0.02 \%, \mathrm{w} / \mathrm{v}$, glucose, $0.04 \%$, w/v, yeast extract) to early exponential phase $\left(\mathrm{OD}_{660} 0 \cdot 3\right)$. Subsequently, glucose was added to a final concentration of $2 \%(\mathrm{w} / \mathrm{v})$. Samples for total RNA isolation were taken at the times indicated in the figures. Nitrogen starvation/refeeding was done as follows. Exponentially growing, prototrophic RG7M1 cells were transferred to nitrogen starvation medium $(0.7 \%, w / v, Y N B$ without amino acids and ammonium sulphate). After overnight starvation, ammonium sulphate was added to a final concentration of $30 \mathrm{mM}$. Samples for total RNA isolation were taken at the times indicated in Fig. 4.

Construction of strain CPL25. In strain CPL25 the LYS2 gene is put under transcriptional control of the L25 rp-gene promoter. To this end, a $0.5 \mathrm{~kb}$ fragment of the upstream region (UR) of the rp-gene L25, derived from plasmid pBMCY138 (Leer et al., 1984), was fused to the EcoRV site present in the leader of LYS2, derived from plasmid pDP5 (Fleig et al., 1986; a generous gift from P. Philippsen). This L25 UR contains the major transcription initiation sites and also the natural upstream activation region (consisting of two Rap1-binding sites around -400 ) of L25. In front of the pL25-LYS2 fusion, we placed the UR of LYS2, a $275 \mathrm{bp} \mathrm{XbaI-EcoRV} \mathrm{fragment} \mathrm{from} \mathrm{plasmid}$ pDP5. This UR (which contains the promoter region of LYS2) and the LYS 2 coding region were used for double homologous recombination, in order to replace the promoter of LYS2, in the yeast genome, by the $L 25 \mathrm{rp}$-gene promoter. To prevent readthrough of transcription derived from the LYS 2 promoter into the pL25-LYS2 fusion, we placed the L25 transcription

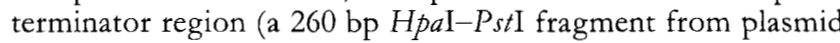
pBMCY138; Leer et al., 1984) upstream of the pL.25-LYS2 fusion. This DNA construct was transformed as a linear fragment to yeast strain CH2881-1D (Fleig et al., 1986) and we selected transformants for lysine prototrophy. This resulted in strain CPL25. Integration into the yeast genome was checked by Southern analysis (results not shown).

Isolation and characterization of glucose response deficient (grd) mutants. Selection of $g r d$ mutants, displaying low expression of the LYS2 reporter gene, was achieved on plates containing $\alpha$-aminoadipate ( $\alpha \mathrm{AA})$. We determined that the 
optimal growth medium for selection was composed of: $0 \cdot 167 \%$ (w/v) Y ${ }^{\prime} \mathrm{NB}$ (without amino acids and ammonium sulphate), $2 \%(\mathrm{w} / \mathrm{v})$ glucose, $1.5 \%(\mathrm{w} / \mathrm{v})$ agarose, $8 \mathrm{mg}$ leucine $\mathrm{l}^{-1}, 4 \mathrm{mg}$ uracil $\mathrm{l}^{-1}, 4 \mathrm{mg}$ adenine $\mathrm{l}^{-1}$, $13 \mathrm{mg}$ tryptophan $\mathrm{l}^{-1}, 5 \mathrm{mg} \alpha$ aminoadipate $\mathrm{ml}^{-1}$. CPL25 cells were plated on this selection medium and mutagenized by UV irradiation. The dose of radiation was set to give $10-20 \%$ survival of yeast cells as compared to untreated cells. Colonies with $\alpha$-aminoadipateresistant yeast cells were subsequently grown on glycerol- and glucose-based medium. Mutants were selected which displayed normal growth on glycerol, but decreased growth on glucose, as compared to wild-type. The rationale for this additional screening was that one may expect that yeast cells with a defect in the glucose upshift response of rp-gene transcription will display reduced growth on glucose. By using this step we also prevented isolation of petite mutants and mutants with a nonspecific growth defect. With the positive yeast mutants remaining after the second selection step, a glucose upshift experiment was performed and Northern analysis was done to estimate rp-mRNA levels. Complementation analysis of grdt was carried out making use of a YCP50-based yeast genomic library cloned on a single-copy vector (Rose \& Fink, 1987; a generous gift from W. Kos and H. Tabak, Academical Medical Centre, University of Amsterdam).

Preparation and Northern analysis of RNA. RNA was isolated from yeast cells disrupted with glass beads, essentially as described by Bromley et al. (1982). For the electrophoretic separation of RNA, formaldehyde/agarose gels were used, according to the method of Ausubel et al. (1994). Hybridization was carried out with probes corresponding to the coding regions of the genes encoding the ribosomal proteins L25 and S33 and part of the actin gene, which was used as internal standard. Probes were radioactively labelled by using the Primea-Gene labelling system (Promega). Radioactive signals on the filters were visualized by making use of either a phosphoimager (Molecular Dynamics 425) or films (Kodak, X-omat S).

\section{RESULTS}

\section{Isolation of yeast mutants deficient in the glucose upshift response of rp-gene transcription}

As part of our studies on the mechanisms involved in nutritional control of rp-gene expression in yeast, we attempted to isolate mutants showing defects in the glucose upshift response of rp-gene transcription. To this end we developed a novel selection system, based on the following principle. A yeast strain (CPL25) was constructed, in which the LYS2 gene was put under control of the L25 rp-gene promoter. Northern analysis demonstrated that the resulting fusion gene displayed the pattern of regulation typical for rp-genes (results not shown). Lys $2 p$ converts $\alpha$-aminoadipate into $\alpha$-aminoadipate- $\delta$ semialdehyde which, above a certain threshold, is toxic to yeast cells (Winston \& Bhattacharjee, 1982; Zaret \& Sherman, 1985). Addition of excess amounts of $\alpha$ aminoadipate to the medium may therefore prevent growth. We determined the experimental conditions under which no growth of CPL25 on glucose occurred and then selected for $\alpha$-aminoadipate-resistant CPL25 mutants, in which expression of the reporter LYS 2 driven by the rp-gene promoter should be reduced (see Methods for experimental details). A number of grd (glucose response deficient) mutants were isolated which displayed

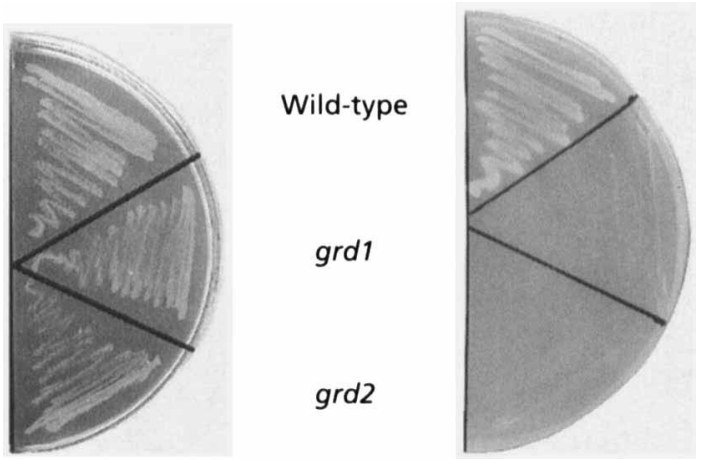

Fig. 1. Growth characteristics of two grd mutants as compared to wild-type cells. Yeast cells on the left-hand plate were grown for $6 \mathrm{~d}$ on YNB medium supplemented with $5 \%$ glycerol; those on the right-hand plate were grown for $2 d$ on YNB medium supplied with glucose.

strongly reduced growth on glucose, but grew normally on glycerol (Fig. 1). With these mutants an upshift experiment was performed by adding glucose in fermentable amounts to glycerol-grown cells. The levels of rpgene mRNA were determined by Northern hybridization (Fig. 2). Surprisingly, the immediate upshift response after addition of glucose appeared not to be affected. However, in grd mutants the initial increase of $\mathrm{rp}-\mathrm{mRNA}$ levels was followed by a strong decline 60-90 min after the shift, whereas in wild-type cells a threefold increase was maintained for at least $180 \mathrm{~min}$ (compare Fig. 2b and 2c with Fig. 2a). We have only further investigated mutant grd1.

The poor growth of mutant grd1 on glucose allowed us to carry out a complementation analysis. grd1 was transformed with a single-copy yeast genomic library and transformants were selected that displayed wild-type growth on glucose-containing plates. In addition, these transformants exhibited a normal glucose upshift response of rp-gene transcription as measured by Northern analysis (result not shown). Plasmids isolated from a number of different transformants were all found to contain overlapping inserts, indicating that in all cases the suppression was due to the same gene. Further characterization of the inserts revealed that they contain HIS7, a gene encoding an amidotransferase: cyclase which catalyses the fifth and sixth step of the histidine biosynthetic pathway (Kuenzler et al., 1993). Consistent with this finding, the growth defect of grd1 on glucose-containing medium could be restored by addition of histidine (result not shown). Thus growth of grdt on glucose appears to be affected due to limited production of histidine. The initial activation of rp-gene transcription observed in this mutant, despite its impaired growth on glucose, suggests that this initial activation occurs independently of the growth potential of the yeast cell (we define here growth potential operationally as the nutritional condition which ensures a certain growth rate). We hypothesize that the subsequent decrease in rp-mRNA levels most probably reflects the lack of sufficient cellular protein-biosynthetic capacity. 
(a)

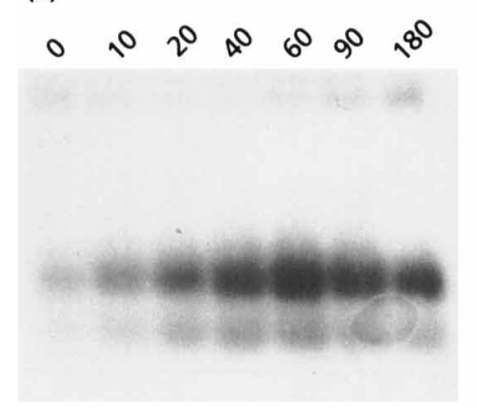

(b)

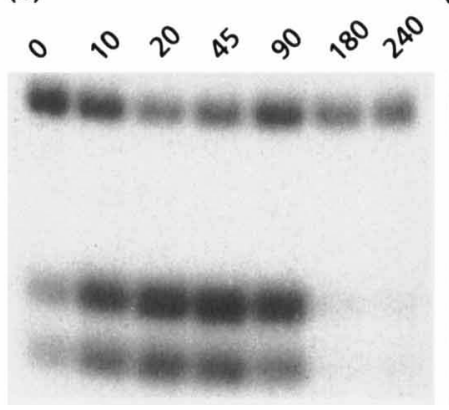

(c)

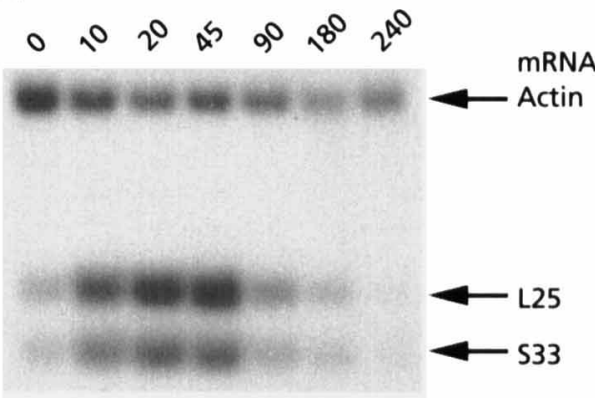

Fig. 2. Northern analysis of rp-mRNA levels upon a glucose upshift performed with strains CPL25 (a), grd1 (b) and grd2 (c). Sampling times ( $\mathrm{min}$ ) are indicated above the respective lanes in Figs $2,3,4 a, b$ and 5.

(a)

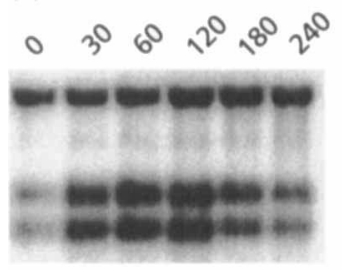

(b)

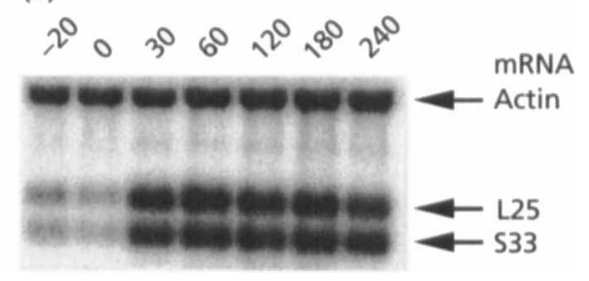

Fig. 3. Northern analysis of rp-mRNA levels upon a glucose upshift performed with strain Y153. The cells were pre-grown in YNB/glycerol medium and divided into two portions. With each portion a glucose upshift was carried out in the absence (a) or presence (b) of histidine [histidine was added $20 \mathrm{~min}(t=-20)$ before the addition of glucose $(t=0)$ ].
Further evidence that the initial upshift response can occur in the absence of an adequate growth potential was obtained using strain Y153, in which the HIS3 gene has been placed under control of an artificial promoter consisting of several Gal4p-binding sites, resulting in a constitutive low level of HIS 3 transcription (Durfee $e t$ al., 1993). In the presence of $10 \mathrm{mM} \mathrm{3}$-aminotriazole, a competitive inhibitor of His $3 \mathrm{p}$, histidine production is limiting for growth of Y153 on glucose but not on glycerol, resulting in approximately equal growth rates on the two carbon sources $\left(\mu=0.054 \mathrm{~h}^{-1}\right.$ and $0.057 \mathrm{~h}^{-1}$, respectively). A nutritional upshift experiment was performed with strain Y153 and rp-mRNA levels were measured by Northern hybridization. The results (Fig. 3a) again show an initial increase in rp-mRNA levels followed by a drop to the starting value after $120 \mathrm{~min}$. This decrease did not occur when histidine was added $20 \mathrm{~min}$ before the addition of glucose (Fig. 3b). These data are fully consistent with the results presented above for $\mathrm{grd} 1$, and support the hypothesis that the upshift response consists of two phases: an initial, rapid upshift response, which is independent of the growth potential, followed by a sustained response, in which rp-mRNA levels are adjusted to the actual growth demands.

\section{The initial upshift response requires nitrogen in addition to glucose}

We subsequently examined the upshift response of yeast cells starved on glycerol medium for nitrogen or for an amino acid, both conditions where addition of glucose cannot stimulate the growth rate. As shown in Fig. 4(a) $\left(t=0^{\prime}\right)$, overnight starvation for histidine severely dimin- ished rp-gene transcription and subsequent addition of glucose led to a strong, but only transient, increase. In contrast, cells starved for nitrogen on a non-fermentable carbon source did not display the initial glucose upshift response (Fig. 4b). However, we did observe a strong increase in rp-gene transcription upon addition of ammonium sulphate $240 \mathrm{~min}$ after the addition of glucose. Apparently, the rapid initial activation of rp-gene transcription upon a nutritional upshift requires at least the presence of an adequate nitrogen source in addition to glucose (or in general perhaps a fermentable carbon source). Consistent with this notion, refeeding with nitrogen of cells starved for nitrogen on a glycerolcontaining medium did not result in a significant activation of rp-gene transcription (Fig. 4c).

\section{The sustained response does not require Pka activity}

Previously, evidence has been obtained that rp-gene transcription is regulated by Pka (Griffioen et al., 1994; Klein \& Struhl, 1994; Neuman-Silberberg et al., 1995). On the other hand, we found that the increase in rp-gene transcription after addition of glucose does not depend on the transient elevation of intracellular cAMP levels (Kraakman et al., 1993). To investigate the involvement of $\mathrm{Pka}$ in the initial and the sustained upshift response, a glucose upshift was performed using a Pka null mutant yeast strain (SGP406) in which the three genes encoding the functionally redundant catalytic subunits of $\mathrm{Pka}$ (TPK1-3) are deleted. In addition, YAK1 is deleted to suppress the growth deficiency caused by the deletion of TPK1-3 alone (Garrett \& Broach, 1989). Unexpectedly, addition of glucose to this $\mathrm{Pka}$ null mutant strain, grown 
(a)

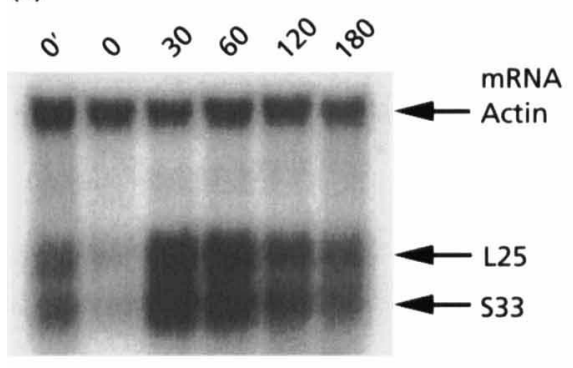

(b)

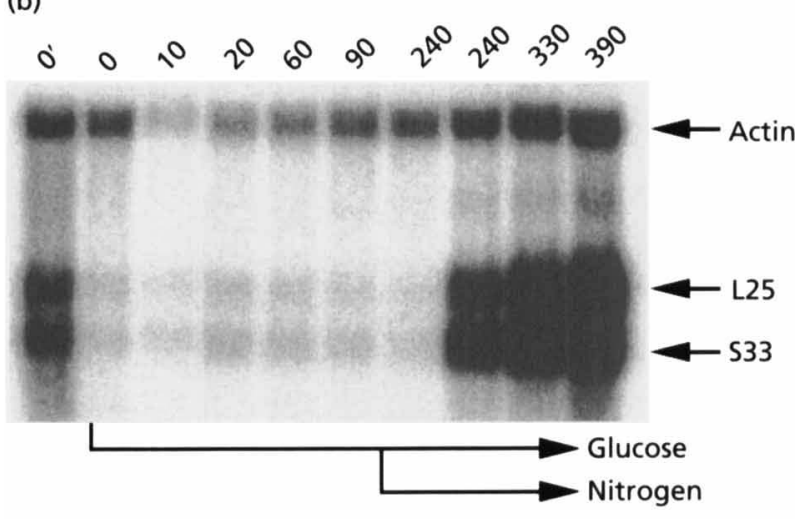

(c)

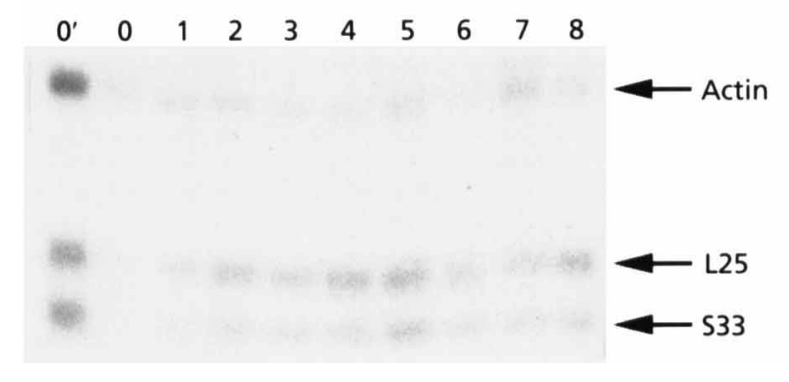

Fig. 4. (a) Northern analysis of rp-mRNA levels upon a glucose upshift performed with histidine-starved yeast cells. JT100 cells were pre-grown on $\mathrm{YNB}$ /glycerol medium $\left(t=0^{\prime}\right)$, transferred to $\mathrm{YNB} / \mathrm{glycerol}$ medium without histidine and then starved overnight before the addition of glucose $(t=0)$. (b, c) Northern analysis of rp-mRNA levels upon a nitrogen starvation/refeeding experiment with prototrophic strain RG7M1 (see Methods for details). At $t=0$ glucose (b) or ammonium sulphate (c) was added to nitrogen-starved yeast cells, which were grown on glycerol. In (b) ammonium sulphate was also added at $t=240 \mathrm{~min}$. In (c) sampling times are indicated in hours.

on glycerol, led to elevated levels of rp-mRNA (Fig. 5a). However, the increase was significantly delayed compared to that of wild-type cells (cf. Fig. 2a), which usually show a strong increase of $\mathrm{mRNA}$ levels within $20 \mathrm{~min}$. Quantification of the signals confirmed these observations (data not shown). These results indicate that $\mathrm{Pka}$ is dispensable for the long-term nutritional regulation of rp-gene transcription, and imply that the molecular mechanisms underlying this delayed response do not depend on Pka.

To investigate whether the observed Pka-independent stimulation of rp-gene transcription concerns the sustained response only, we performed a glucose upshift with the Pka null mutant in the presence of iodoacetamide (Fig. $5 c$ ). Iodoacetamide is a non-competitive inhibitor of the glycolytic enzyme glyceraldehyde-3-phosphate dehydrogenase, and thus abolishes growth on both glycerol and glucose. The results in Fig. 5(c) show that the addition of iodoacetamide to strain SGP406 prevented any increase of rp-gene mRNA levels upon addition of glucose. On the other hand, a carbon source upshift with wild-type cells in the presence of this drug caused a strong activation of rpgene transcription that was, however, not sustained (Fig. $5 b)$. This latter result provides further evidence that glucose triggers the transient, initial upshift response even when the cellular growth potential is not increased. The absence of the initial nutritional upshift response in this Pka null mutant also supports the conclusion that this initial response is Pka-dependent. Furthermore, we conclude that the observed Pka-independent increase in $\mathrm{rp}$ mRNA levels upon the addition of glucose in this particular mutant corresponds to the sustained, growthdependent upshift response.

\section{DISCUSSION}

In this paper we describe the isolation of yeast mutants deficient in glucose upshift regulation of rp-gene transcription, making use of a novel mutant selection system. This strategy resulted in mutants showing strongly reduced growth on glucose. We established that the impaired growth of one of these mutants, grd1, on glucose was due to limited production of histidine. Unexpectedly, these grd mutants displayed a normal transient increase of rp-gene transcription directly after the addition of glucose, but this was followed by a strong decrease of $\mathrm{rp}$ mRNA levels. These data suggest that the glucose upshift response of $\mathrm{rp}$-gene transcription consists of two phases: an initial phase, independent of the growth potential, followed by a sustained phase which is dependent on the ability to grow.

Further experimental data consistent with this hypothesis were obtained. First, such an initial upshift of rp-gene transcription was also found in yeast cells displaying reduced growth on glucose due to the production of limited amounts of histidine (Fig. 3a). Secondly, a similar observation was made when yeast cells were starved for histidine (Fig. 4a). Thirdly, yeast cells in which glycolysis is blocked also display an initial glucose upshift response of rp-gene transcription (Fig. 5b). Fourthly, making use of different sets of $h \times k 1 / 2$ glk 1 mutants, evidence was obtained that phosphorylation of the sugar is not required to activate rp-gene transcription upon addition of glucose to glycerol-grown cells (Pernambuco tt al., 1996). These results taken together demonstrate that glucose, when added to a respiring yeast culture, may enhance rp-gene transcription irrespective of whether this addition leads to an actual growth improvement, provided that an adequate nitrogen source is available. Furthermore, we conclude that the underlying mechanism which activates rp-gene transcription directly after a glucose upshift is funda- 
(a)

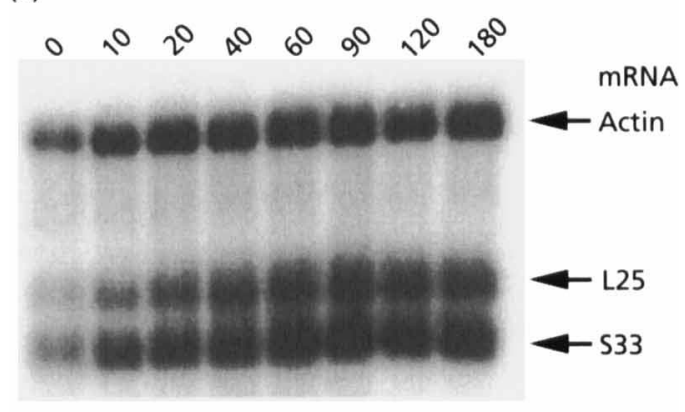

(b)

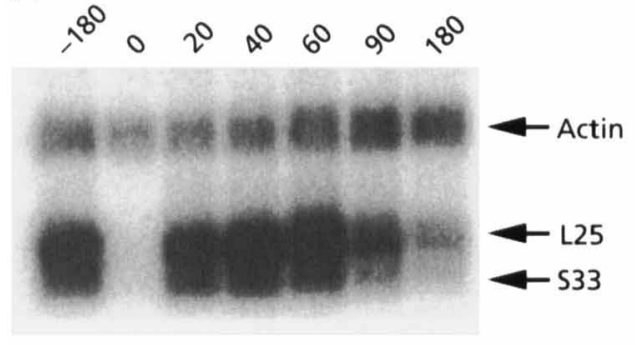

(b)

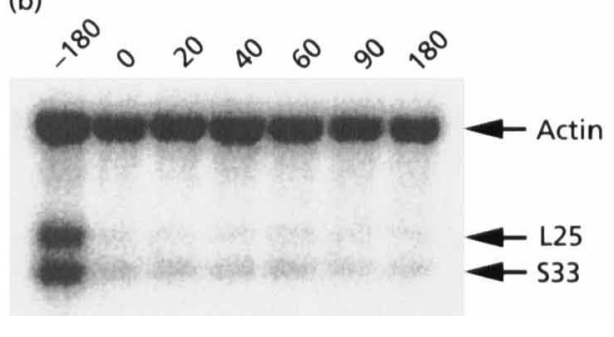

Fig. 5. Northern analysis of rp-mRNA levels upon a glucose upshift performed with wild-type strain CPL25 (b) and the Pka null mutant SGP406 $(a, c)$. In (b) and (c) iodoacetamide was added $(t=-180)$ prior to the addition of glucose $(t=0)$.

mentally different from that of steady-state growing yeast cells. In the latter case rp-gene transcription is correlated with the growth demands (Kraakman et al., 1993). The finding discussed above also explains why we have isolated grd mutants deficient in the sustained phase of the glucose upshift response, because every mutation affecting general metabolism would cause a reduced level of rp-gene transcription in the sustained phase of the upshift response. Since the initial response is transient, while the screening was performed under steady-state conditions, the selection could only score defects in the sustained response.

The results described in this paper provide evidence that the initial, growth-potential-independent response is initiated by activation of Pka. In Fig. 5(c) it is demonstrated that the initial glucose upshift regulation of rp-gene transcription is totally absent in the Pka null mutant. Consistently, also a tpk $1^{w 1} t p k .2 t p k 3$ bcy 1 mutant does not show the initial response (Griffioen et al., 1994; Klein \& Struhl, 1994). Recently it was demonstrated that the effect of amino acid starvation on rp-gene transcription is

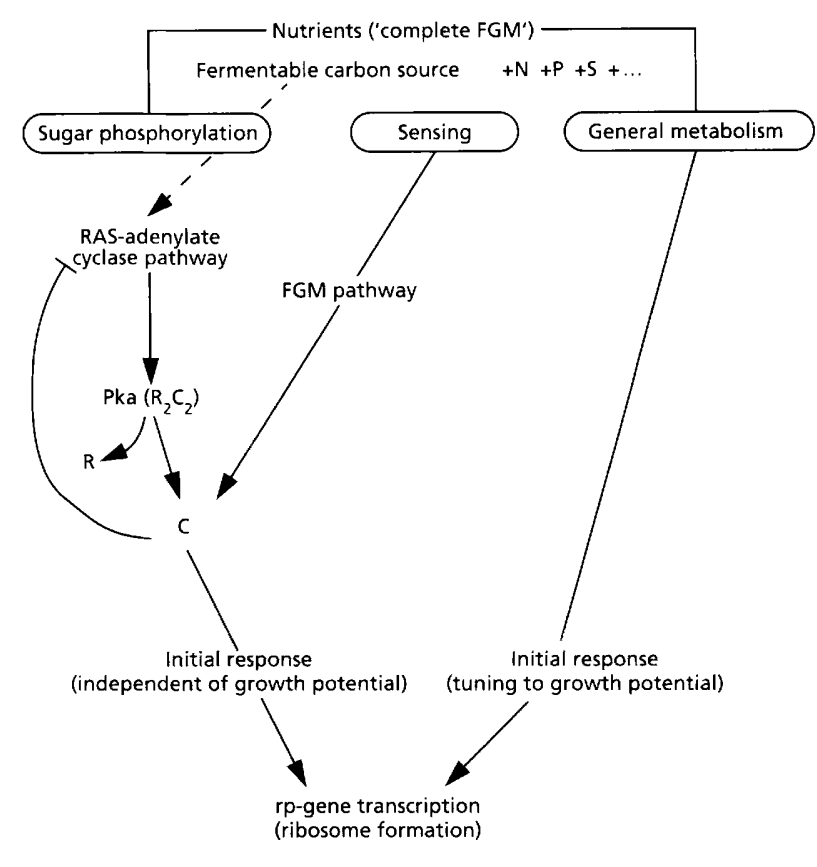

Fig. 6. Working model for the nutritional regulation of rp-gene transcription in $S$. cerevisiae. Nutritional upshift regulation of rp-gene transcription comprises two phases: an initial response and a sustained response. Pka may be predominantly involved in the initial upshift response. Activation of $\mathrm{Pka}$ is regulated by both the RAS/adenylate cyclase pathway and the FGM pathway (see text). Activation of the RAS/adenylate cyclase pathway requires phosphorylation of the sugar. Furthermore, this pathway is subjected to feedback regulation (Nikawa et al., 1987). The FGM pathway is only activated by a complete growth medium supplemented with a fermentable sugar. The sustained response occurs in the absence of $\mathrm{Pka}$, and is dependent on the ability of cells to grow. We hypothesize that this sustained response correlates rp-gene transcription with the actual growth potential.

suppressed in a mutant displaying high, constitutive activity of Pka activity (Klein \& Struhl, 1994). In agreement with this, we demonstrate in this paper that yeast cells, when starved for histidine, transiently activate rp-gene transcription upon addition of glucose. These data also support the model in which the initial response of rp-gene transcription is brought about through activation of Pka.

We have previously demonstrated that the Pka-initiated glucose upshift regulation of rp-gene transcription does not require the transient, glucose-induced increase in cAMP production, and indeed can occur in cAMP-starved cells (Griffioen et al., 1994; Kraakman et al., 1993). Recently, additional evidence was obtained demonstrating that the nutritional upshift response is not mediated exclusively by the RAS/adenylate cyclase pathway. First, the addition of nitrogen to nitrogen-starved cells on glucose-containing medium led to a strong activation of rp-gene transcription (Neuman-Silberberg et al., 1995; this paper), which cannot be the result of an increase in cAMP levels, because the RAS/adenylate cyclase pathway 
is glucose repressed (Arguelles et al., 1990; Beullens et al., 1988). Secondly, it was found that upshift regulation of rp-gene transcription takes place in mutants unable to phosphorylate the sugar (Pernambuco et al., 1996.). Since for activation of the RAS/adenylate cyclase pathway phosphorylation of the sugar is required (Beullens $e t$ al., 1988), this result further underlines that another pathway must be involved in the upshift response. From these data we conclude that cAMP is not exclusively required for the glucose upshift response of rp-gene transcription but may serve as a 'booster' of Pka activity. This may result in a very rapid and strong activation of rp-gene transcription, particularly during the transition from non-fermentable to fermentable growth. Besides the RAS/adenylate cyclase pathway an additional pathway appears to be involved in the initial upshift response of rp-gene transcription. A likely candidate for this alternative pathway is the recently defined FGM-induced pathway. It has been proposed that this pathway further stimulates the free catalytic subunits of Pka and may be implicated in the control of a wide variety of processes, such as regulation of transcription of stress-related genes and the posttranslational modification of enzymes involved in energy mobilization (reviewed by Thevelein, 1994). Importantly, activation of this pathway can only be achieved by a complete growth medium supplemented with a fermentable carbon source. In this paper we indeed show that the initial response does not take place under all conditions of nutrient limitation. When cells were starved for nitrogen on glycerol-containing medium, the initial glucose upshift response of rp-gene transcription appeared to be absent. Also the other way around, when we re-fed nitrogen to nitrogen-starved cells on glycerolcontaining medium, rp-gene transcription did not increase significantly. Apparently, in order to generate this response at least nitrogen in addition to glucose (but most probably a complete growth medium) are required. On the basis of the results described so far we propose a working model in which both the RAS/adenylate cyclase pathway and the FGM pathway mediate the initial glucose upshift response (Fig. 6). Apart from this transient, growth-potential-independent response, we have observed that under conditions of growth limitation in the course of the experiment rp-gene mRNA levels are strongly decreased.

After the initial response rp-gene transcription might be linked to the actual demand for protein biosynthesis capacity, which is, of course, dictated by the growth conditions. This is demonstrated in Fig. 3(a). Here, the demand for protein biosynthetic capacity is not increased after the addition of glucose, due to the growth-limiting amount of histidine, and therefore the transiently increased rp-mRNA levels return to the starting levels. In addition, we have shown that the sustained, growthpotential-dependent response does not require Pka by performing a glucose upshift experiment using a tpk1-3 yak1 mutant (Fig. 5a). From these results we conclude that the underlying mechanism of the sustained response is different from that of the initial response and is probably somehow determined by ongoing metabolism (see Fig. 6).
Another indication that more than one pathway is involved in regulating rp-gene transcription is that a wimp mutant $\left(t p k^{w} b c y 1\right)$ displayed normal growth on glucose compared with wild-type (results not shown), although this mutant does not show the initial phase of the glucose upshift response of $\mathrm{rp}$-gene transcription (Griffioen et al., 1994; Klein \& Struhl, 1994). If mRNA levels remained low after the addition of glucose, one may expect a decreased growth rate due to a limited protein biosynthetic capacity.

In wild-type cells, the initial response and the sustained response are probably not strictly separated but their contribution may depend on the environmental stimuli. When cells are grown in a steady-state condition the Pkaindependent, growth-potential-dependent pathway may be involved predominantly in maintaining appropriate rpmRNA levels, whereas directly after a carbon source upshift, Pka activity is most important for the activation of rp-gene transcription. Because $\mathrm{Pka}$ is involved in the regulation of many growth-related processes, this will also have a major impact on metabolism in general. In this way metabolism is directly regulated by the presence of nutrients.

\section{ACKNOWLEDGEMENTS}

The authors are indebted to J. Thevelein, P. Philippsen and J. Elledge for providing strains and plasmids and to P. Gonçalves and M. Bosman, who also contributed to this work. They thank Professor H. A. Raué for critical reading of the manuscript.

\section{REFERENCES}

Arguelles, J. C., Mbonyi, K., Van Aelst, L., Vanhalewyn, M., Jans, A. W. \& Thevelein, J. M. (1990). Absence of glucose-induced cAMP signaling in the Saccharomyces cerevisiae mutants cat 1 and cat 3 which are deficient in derepression of glucose-repressible proteins. Arch Microbiol 154, 199-205.

Ausubel, F. M., Brent, R., Kingston, R. E., Moore, D. D., Seidman, J. G., Smith, J. A. \& Struhl, K. (1994). Current Protocols in Molecular Biology, vol. 1. New York: John Wiley.

Belazzi, T., Wagner, A., Wieser, R., Schanz, M., Adam, G., Hartig, A. \& Ruis, H. (1991). Negative regulation of transcription of the Saccharomyces cerevisiae catalase $\mathrm{T}(C T T 1)$ gene by cAMP is mediated by a positive control element. EMBO J 10, 585-592.

Beullens, M., Mbonyi, K., Geerts, L., Gladines, D., Detremerie, K., Jans, A. W. \& Thevelein, J. M. (1988). Studies on the mechanism of the glucose-induced cAMP signal in glycolysis and glucose repression mutants of the yeast Saccharomyces cerevisiae. Eur J Biochem 172, 227-231.

Broach, J. R. (1991). RAS genes in Saccharomyces cerevisiae: signal transduction in search of a pathway. Trends Genet 7, 28-33.

Broek, D., Toda, T., Michaeli, T., Levin, L., Birchmeier, C., Zoller, M., Powers, S. \& Wigler, M. (1987). The S. cerevisiae CDC25 gene product regulates the RAS/adenylate cyclase pathway. Cell 48, 789-799.

Bromley, S., Hereford, L. \& Rosbash, M. (1982). Further evidence 
that the rna2 mutation of Saccharomyces cerevisiae affects mRNA processing. Mol Cell Biol 2, 1205-1211.

Cameron, S., Levin, L., Zoller, M. \& Wigler, M. (1988). cAMPindependent control of sporulation, glycogen metabolism, and heat shock resistance in $S$. cerevisiae. Cell 53, 555-566.

Donovan, D. M. \& Pearson, N. J. (1986). Transcriptional regulation of ribosomal proteins during a nutritional upshift in Saccharomyces cerevisiae. Mol Cell Biol 6, 2429-2435.

Durfee, T., Becherer, K., Chen, P. L., Yeh, S. H., Yang, Y., Kilburn, A. E., Lee, W. H. \& Elledge, S. J. (1993). The retinoblastoma protein associates with the protein phosphatase type 1 catalytic subunit. Genes Dev 7, 555-569.

Durnez, P., Pernambuco, M. B., Oris, E., Arguelles, J. C., Mergelsberg, H. \& Thevelein, J. M. (1994). Activation of trehalase during growth induction by nitrogen sources in the yeast Saccharomyces cerevisiae depends on the free catalytic subunits of cAMP-dependent protein kinase, but not on functional Ras proteins. Yeast 10, 1049-1064.

Fleig, U. N., Pridmore, R. D. \& Philippsen, P. (1986). Construction of LYS 2 cartridges for use in genetic manipulations of Saccharomyces cerevisiae. Gene 46, 237-245.

Gancedo, J. M. (1992). Carbon catabolite repression in yeast. Eur J Biochem 206, 297-313.

Garrett, S. \& Broach, J. (1989). Loss of Ras activity in Saccharomyces cerevisiae is suppressed by disruptions of a new kinase gene, $Y A K 1$, whose product may act downstream of the cAMP-dependent protein kinase. Genes Dev 3, 1336-1348.

Goncalves, P. M., Griffioen, G., Minnee, R., Bosma, M., Kraakman, L. S., Mager, W. H. \& Planta, R. J. (1995). Transcription activation of yeast ribosomal protein genes requires additional elements apart from binding sites for Abf1p or Rap1p. Nucleic Acids Res 23, 1475-1480.

Griffioen, G., Mager, W. H. \& Planta, R. J. (1994). Nutritional upshift response of ribosomal protein gene transcription in Saccharomyces cerevisiae. FEMS Microbiol Lett 123, 137-144.

Herruer, M. H., Mager, W. H., Woudt, L. P., Nieuwint, R. T., Wassenaar, G. M., Groeneveld, P. \& Planta, R. J. (1987). Tran scriptional control of yeast ribosomal protein synthesis during carbon-source upshift. Nucleic Acids Res 15, 10133-10144.

Hinnebusch, A. G. (1988). Mechanisms of gene regulation in the general control of amino acid biosynthesis in Saccharomyces cerevisiae. Microbiol Rev 52, 248-273.

Hinnebusch, A. G. (1994). Translational control of GCN4: an in vivo barometer of initiation-factor activity. Trends Biochem Sci 19, 409-414.

Hirimburegama, K., Durnez, P., Keleman, J., Oris, E., Vergauwen, R., Mergelsberg, H. \& Thevelein, J. M. (1992). Nutrient-induced activation of trehalase in nutrient-starved cells of the yeast Saccharomyces cerevisiae: CAMP is not involved as second messenger. J Gen Microbiol 138, 2035-2043.

Kataoka, T., Powers, S., McGill, C., Fasano, O., Strathern, J., Broach, J. \& Wigler, M. (1984). Genetic analysis of yeast RAS1 and R.AS2 genes. Cell 37, 437-445.

Kief, D. R. \& Warner, J. R. (1981). Coordinate control of syntheses of ribosomal ribonucleic acid and ribosomal proteins during nutritional shift-up in Saccharomyces cerevisiae. Mol Cell Biol $\mathbf{1}$, 1007-1015.

Klein, C. \& Struhl, K. (1994). Protein kinase A mediates growthregulated expression of yeast ribosomal protein genes by modulating Rap1 transcriptional activity. Mol Cell Biol 14, 1920-1928.

Kraakman, L. S., Griffioen, G., Zerp, S., Groeneveld, P., Thevelein,
J. M., Mager, W. H. \& Planta, R. J. (1993). Growth-related expression of ribosomal protein genes in Saccharomyces cerevisiae. Mol Gen Genet 239, 196-204.

Kuenzler, M., Balmelli, T., Egli, C. M., Paravicini, G. \& Braus, G. H. (1993). Cloning, primary structure, and regulation of the HIS 7 gene encoding a bifunctional glutamine amidotransferase: cyclase from Saccharomyces cerevisiae. J Bacteriol 175, 5548-5558.

Leer, R. J., van Raamsdonk-Duin, M. M., Hagendoorn, M. J., Mager, W. H. \& Planta, R. J. (1984). Structural comparison of yeast ribosomal protein genes. Nucleic Acids Res 12, 6685-6700.

Mager, W. H. \& Planta, R. J. (1991). Coordinate expression of ribosomal protein genes in yeast as a function of cellular growth rate. Mol Cell Biochem 104, 181-187.

Marchler, G., Schuller, C., Adam, G. \& Ruis, H. (1993). A Saccharomyces cerevisiae UAS element controlled by protein kinase $A$ activates transcription in response to a variety of stress conditions. EMBO J 12, 1997-2003.

Matsumoto, K., Uno, I. \& Ishikawa, T. (1983a). Control of cell division in Saccharomyces cerevisiae mutants defective in adenylate cyclase and cAMP-dependent protein kinase. Exp Cell Res 146, $151-161$

Matsumoto, K., Uno, I. \& Ishikawa, T. (1983b). Initiation of meiosis in yeast mutants defective in adenylate cyclase and cyclic AMP-dependent protein kinase. Cell 32, 417-423.

Neuman-Silberberg, F. S., Bhattacharya, S. \& Broach, J. R. (1995). Nutrient availability and the RAS/cyclic AMP pathway both induce expression of ribosomal protein genes in Saccharomyces cerevisiae but by different mechanisms. Mol Cell Biol 15, 3187-3196.

Nikawa, J., Cameron, S., Toda, T., Ferguson, K. M. \& Wigler, M. (1987). Rigorous feedback control of cAMP levels in Saccharomyces cerevisiae. Genes Dev 1, 931-937.

Pernambuco, M. B., Winderickx, J., Crauwels, M., Griffioen, G., Mager, W. H. \& Thevelein, J. M. (1996). Glucose-triggered signalling in Saccharomyces cerevisiae: different requirements for sugar phosphorylation between cells grown on glucose and those grown on non-fermentable carbon sources. Microbiology 142, 1775-1782

Rose, M. D. \& Fink, G. R. (1987). $K A R 1$, a gene required for function of both intranuclear and extranuclear microtubules in yeast. Cell 48, 1047-1060.

Tanaka, K., Matsumoto, K. \& Toh-e, A. (1988). Dual regulation of the expression of the polyubiquitin gene by cyclic AMP and heat shock in yeast. EMBO J 7, 495-502.

Thevelein, J. M. (1991). Fermentable sugars and intracellular acidification as specific activators of the RAS-adenylate cyclase signalling pathway in yeast: the relationship to nutrient-induced cell cycle control. Mol Microbiol 5, 1301-1307.

Thevelein, J. M. (1992). The RAS-adenylate cyclase pathway and cell cycle control in Saccharomyces cerevisiae. Antonie Leewwenboek, 62, 109-130.

Thevelein, J. M. (1994). Signal transduction in yeast. Yeast 10, 1753-1790.

Toda, T., Cameron, S., Sass, P., Zoller, M., Scott, J. D., McMullen, B., Hurwitz, M., Krebs, E. G. \& Wigler, M. (1987a). Cloning and characterization of $B C Y 1$, a locus encoding a regulatory subunit of the cyclic AMP-dependent protein kinase in Saccharomyces cerevisiae. Mol Cell Biol 7, 1371-1377.

Toda, T., Cameron, S., Sass, P., Zoller, M. \& Wigler, M. (1987b). Three different genes in $S$. cerevisiae encode the catalytic subunits of the cAMP-dependent protein kinase. Cell 50, 277-287.

Toda, T., Uno, I., Ishikawa, T., Powers, S., Kataoka, T., Broek, D., Cameron, S., Broach, J., Matsumoto, K. \& Wigler, M. (1985). In 
yeast, RAS proteins are controlling elements of adenylate cyclase. Cell 40, 27-36.

Trumbly, R. J. (1992). Glucose repression in the yeast Saccharomyces cerevisiae. Mol Microbiol 6, 15-21.

Werner-Washburne, M., Braun, E., Johnston, G. C. \& Singer, R. A.

(1993). Stationary phase in the yeast Saccharomyces cerevisiae. Microbiol Rev 57, 383-401.

Winston, M. K. \& Bhattacharjee, J. K. (1982). Growth inhibition by $\alpha$-aminoadipate and reversal of the effect by specific amino acid supplements in Saccharomyces cerevisiae. J Bacteriol 152, 874-879.

Zaret, K. S. \& Sherman, F. (1985). $\alpha$-Aminoadipate as a primary nitrogen source for Saccbaromyces cerevisiae mutants. J Bacteriol 162, $579-583$.

Received 2 February 1996; revised 13 March 1996; accepted 15 March 1996. 